\title{
Injectivity and Surjectivity of the Dress Map
}

\author{
Ricardo G Rojas-Echenique ${ }^{1}$ \\ Department of Mathematics, Reed College, 3203 SE Woodstock Blvd, Portland, OR 97202, \\ $U S A$
}

\begin{abstract}
For a nontrivial finite Galois extension $L / k$ (where the characteristic of $k$ is different from 2) with Galois group $G$, we prove that the Dress map $h_{L / k}$ : $A(G) \rightarrow G W(k)$ is injective if and only if $L=k(\sqrt{\alpha})$ where $\alpha$ is not a sum of squares in $k^{\times}$. Furthermore, we prove that $h_{L / k}$ is surjective if and only if $k$ is quadratically closed in $L$. As a consequence, we give strong necessary conditions for faithfulness of the Heller-Ormsby functor $c_{L / k}^{*}: \mathrm{SH}_{G} \rightarrow \mathrm{SH}_{k}$, as well as strong necessary conditions for fullness of $c_{L / k}^{*}$.
\end{abstract}

Keywords:

2010 MSC: 11E81; 19A22; 19G99; 14F42; 55P91

\section{Introduction}

Let $k$ be a field of characteristic different from 2 and let $L / k$ be a finite Galois extension with Galois group $G$. Let $A(G)$ denote the Burnside ring of $G$ and let $G W(k)$ denote the Grothendieck-Witt ring of $k$. Recall that, as abelian groups, $A(G)$ is freely generated under disjoint union by cosets $G / H$ where $H$ runs through a set of representatives for conjugacy classes of subgroups, and $G W(k)$ is generated by 1-dimensional quadratic forms $\langle a\rangle$, where $a$ runs through the group of square classes $k^{\times} /\left(k^{\times}\right)^{2}$, under orthogonal sum $\langle a\rangle+\langle b\rangle=\langle a, b\rangle$. Multiplication in $A(G)$ is given by cartesian product with identity $G / G$ and multiplication in $G W(k)$ is given by the Kronecker product $\langle a\rangle\langle b\rangle=\langle a b\rangle$ with identity $\langle 1\rangle$. Following the construction in $[1$, Appendix B], the Dress map $h_{L / k}: A(G) \rightarrow G W(k)$ is a ring homomorphism that takes the coset $G / H$ to the trace form $\operatorname{tr}_{L^{H} / k}\langle 1\rangle_{L^{H}}$, the quadratic form $x \mapsto \operatorname{tr}_{L^{H} / k} x^{2}$. (Our restriction on the characteristic of $k$ is necessary for $h_{L / k}$ to be well defined.)

A particular point of interest is that the Dress map appears naturally in the study of equivariant and motivic stable homotopy theory. Heller and Ormsby $[2$, $\S 4]$ construct a strong symmetric monoidal triangulated functor $c_{L / k}^{*}: \mathrm{SH}_{G} \rightarrow$ $\mathrm{SH}_{k}$ from the stable $G$-equivariant homotopy category to the stable motivic homotopy category over $k$. This functor induces a homomorphism between the

Email address: rojasecr@reed.edu (Ricardo G Rojas-Echenique)

(c) 2016. This manuscript version is made available under the Elsevier user license http://www.elsevier.com/open-access/userlicense/1.0/ 
endomorphism rings of the unit objects in each category, which are in fact $A(G)$ and $G W(k)$, respectively. In [2, Proposition 3.1], Heller and Ormsby show that this homomorphism agrees with $h_{L / k}$. In particular, fullness and faithfulness of $c_{L / k}^{*}$ are obstructed by surjectivity and injectivity of $h_{L / k}$ respectively.

The main goal of this note is to investigate when the Dress map, and thereby $c_{L / k}^{*}$, is injective or surjective. While Heller and Ormsby have resolved the investigation when $h_{L / k}$ is an isomorphism [2, Theorem 3.4], we proceed by examining injectivity and surjectivity separately.

When $L=k$ it is obvious that $h_{L / k}$ is injective. The following theorem gives a complete account of when $h_{L / k}$ is injective in the remaining cases.

Theorem 1. For a finite nontrivial Galois extension $L / k, h_{L / k}$ is injective if and only if $L=k(\sqrt{\alpha})$ where $\alpha \in k^{\times}$is not a sum of squares in $k^{\times}$.

The proof of Theorem 1 is given in $\S 2$. Note that Theorem 1 , taken with $[2$, Proposition 3.1], immediately gives the following corollary.

Corollary 2. If $c_{L / k}^{*}$ is faithful, then either $L / k$ is the trivial extension or of the form described in Theorem 1.

The following theorem gives a complete account of when the Dress map is surjective.

Theorem 3. For a finite Galois extension $L / k, h_{L / k}$ is surjective if and only if $k$ is quadratically closed in $L$.

The proof of Theorem 3 is given in $\S 3$. The following corollary is immediate.

Corollary 4. If $c_{L / k}^{*}$ is full, then $L / k$ is of the form described in Theorem 3.

Theorems 1 and 3 combine to replicate Heller and Ormsby's result that for a finite Galois extension $L / k, h_{L / k}$ is an isomorphism if and only if either $k$ is quadratically closed and $L=k$, or $k$ is euclidean and $L=k(i)$. If $L / k$ is the trivial extension then Theorem 3 requires that $k$ be quadratically closed, otherwise Theorem 1 requires that $L=k(\sqrt{\alpha})$ and $k^{\times} /\left(k^{\times}\right)^{2}$ contains an element that is not a sum of squares. In the latter case, $k$ must be formally real and then Theorem 3 requires that $k^{\times} /\left(k^{\times}\right)^{2}=\left\{\left(k^{\times}\right)^{2}, \alpha\left(k^{\times}\right)^{2}\right\}$, i.e. $\left|k^{\times} /\left(k^{\times}\right)^{2}\right|=2$, so $k$ is euclidean and $\alpha=-1$.

\section{Proof of Theorem 1}

We begin by stating a number of results that are necessary in the proof of Theorem 1. Many of these results are standard and are stated without proof.

Proposition 5. Let $L / k$ be a finite Galois extension.

1. If $L=k$, then $\operatorname{tr}_{L / k}\langle 1\rangle_{L}=\langle 1\rangle$.

2. If $L=k(\sqrt{\alpha})$, then $\operatorname{tr}_{L / k}\langle 1\rangle_{L}=\langle 2,2 \alpha\rangle$.

3. If $L=k\left(\sqrt{\alpha_{1}}, \sqrt{\alpha_{2}}\right)$, then $\operatorname{tr}_{L / k}\langle 1\rangle_{L}=\left\langle 1, \alpha_{1}, \alpha_{2}, \alpha_{1} \alpha_{2}\right\rangle$. 
The following is a standard result from Galois theory.

Proposition 6. Let $L / k$ be a finite Galois extension with Galois group $G$. If $G \cong \mathbb{Z} / 4 \mathbb{Z}$, then there is a field $E$ between $L$ and $k$ such that $E=k(\sqrt{\alpha})$ where $\alpha=a^{2}+b^{2}$ for some $a, b \in k^{\times}$.

The following theorem is taken directly from Lam [3, Proposition 6.14].

Proposition 7. Let $L / k$ be a finite Galois extension, and let $E$ be any field between $k$ and $L$ with $[L: E]=2 r+1$. Then

$$
t r_{L / k}\langle 1\rangle_{L}=(2 r+1) \operatorname{tr}_{E / k}\langle 1\rangle_{E} .
$$

The following lemma is stated in different terms elsewhere. For a proof of this version see $\S 3$.

Lemma 8. For $\alpha \in k^{\times}$, there are positive integers $a, b$ such that $a\langle 1\rangle=b\langle 2,2 \alpha\rangle$ if and only if $\alpha$ is a sum of squares in $k^{\times}$.

We are now ready to prove Theorem 1 . Suppose that $L=k(\sqrt{\alpha})$ where $\alpha \in k^{\times}$is not a sum of squares in $k^{\times}$. Then the only subgroups of $\operatorname{Gal}(L / k)$ are the trivial subgroup and the entire group. Thus, by Proposition 5, the image of $h_{L / k}$ consists of elements of the form

$$
a\langle 1\rangle+b\langle 2,2 \alpha\rangle \quad \text { where } a, b \in \mathbb{Z} .
$$

Now suppose for contradiction that $h_{L / k}$ is not injective. Then $\operatorname{ker}\left(h_{L / k}\right)$ is nontrivial. That is,

$$
a\langle 1\rangle-b\langle 2,2 \alpha\rangle=0 \quad \text { for some } a, b \in \mathbb{Z}^{+} .
$$

It follows from Lemma 8 that $\alpha$ is a sum of squares in $k^{\times}$. This contradicts the hypothesis so we are done with one direction.

The other direction is more difficult so we separate the proof into lemmas.

Lemma 9. Let $L / k$ be a finite Galois extension with Galois group $G$. If there is an odd prime $p$ such that $p$ divides $|G|$, then $h_{L / k}$ is not injective.

Proof. Suppose there is an odd prime $p$ such that $p$ divides $|G|$. Then by Cauchy's theorem there is a subgroup $H \leq G$ of order $p$, so $\left[L: L^{H}\right]=p$. It follows from Proposition 7 that

$$
\begin{aligned}
h_{L / k}(G / e-p G / H) & =\operatorname{tr}_{L / k}\langle 1\rangle_{L}-p \operatorname{tr}_{L^{H} / k}\langle 1\rangle_{L^{H}} \\
& =p \operatorname{tr}_{L^{H} / k}\langle 1\rangle_{L^{H}}-p \operatorname{tr}_{L^{H} / k}\langle 1\rangle_{L^{H}}=0
\end{aligned}
$$

where $e$ is the trivial subgroup. Clearly, $e$ and $H$ are in distinct conjugacy classes thus no linear combination of $G / e$ and $G / H$ is 0 in $A(G)$. Hence, $\operatorname{ker}\left(h_{L / k}\right)$ is nontrivial so $h_{L / k}$ is not injective.

Lemma 10. Let $L / k$ be a finite Galois extension with Galois group $G$. If $|G|=2^{n}$ for $n>1$ then $h_{L / k}$ is not injective. 
Proof. Suppose $|G|=2^{n}$ for $n>1$. Since a group of order $p^{n}$ has a normal subgroup of order $p^{k}$ for each $0 \leq k \leq n$, G has a normal subgroup $H$ of order $2^{n-2}$. We have $\left|\operatorname{Gal}\left(L^{H} / k\right)\right|=4$, so $\operatorname{Gal}\left(L^{H} / k\right) \cong \mathbb{Z} / 4 \mathbb{Z}$ or $\operatorname{Gal}\left(L^{H} / k\right) \cong$ $\mathbb{Z} / 2 \mathbb{Z} \times \mathbb{Z} / 2 \mathbb{Z}$. We now analyze each case separately.

Suppose $\operatorname{Gal}\left(L^{H} / k\right) \cong \mathbb{Z} / 4 \mathbb{Z}$. Then, by Proposition 6 , there is a subextension $E=k(\sqrt{\alpha})$ of $L$ where $\alpha$ is a sum of two squares. We will use the fact that if $\alpha$ is a sum of two squares, then $2\langle\alpha\rangle=2\langle 1\rangle$ (a more general version of this fact is proved in $\S 3$ ). Now by Proposition 5, and since $\alpha$ is a sum of 2 squares,

$$
\begin{aligned}
h_{L / k}(4 G / G-2 G / \operatorname{Gal}(L / E)) & =4\langle 1\rangle-2 \operatorname{tr}_{E / k}\langle 1\rangle_{E} \\
& =4\langle 1\rangle-2\langle 2,2 \alpha\rangle \\
& =4\langle 1\rangle-4\langle 1\rangle=0 .
\end{aligned}
$$

Clearly $G$ and $\operatorname{Gal}(L / E)$ are in distinct conjugacy classes. Thus $\operatorname{ker}\left(h_{L / k}\right)$ is nontrivial so $h_{L / k}$ is not injective.

Now suppose $\operatorname{Gal}\left(L^{H} / k\right) \cong \mathbb{Z} / 2 \mathbb{Z} \times \mathbb{Z} / 2 \mathbb{Z}$. Then $L^{H}=k\left(\sqrt{\alpha_{1}}, \sqrt{\alpha_{2}}\right)$ and $L^{H}$ has distinct subextensions $E_{1}=k\left(\sqrt{\alpha_{1}}\right), E_{2}=k\left(\sqrt{\alpha_{2}}\right)$, and $E_{3}=k\left(\sqrt{\alpha_{1} \alpha_{2}}\right)$. Let $H_{1}=\operatorname{Gal}\left(L / E_{1}\right), H_{2}=\operatorname{Gal}\left(L / E_{2}\right)$, and $H_{3}=\operatorname{Gal}\left(L / E_{3}\right)$. Consider

$$
\begin{aligned}
& h_{L / k}\left(4 G / G+2 G / H-2 G / H_{1}-2 G / H_{2}-2 G / H_{3}\right) \\
& =4 \operatorname{tr}_{L^{G} / k}\langle 1\rangle_{L^{G}}+2 \operatorname{tr}_{L^{H} / k}\langle 1\rangle_{L^{H}}-2 \operatorname{tr}_{E_{1} / k}\langle 1\rangle_{E_{1}}-2 \operatorname{tr}_{E_{2} / k}\langle 1\rangle_{E_{2}}-2 \operatorname{tr}_{E_{3} / k}\langle 1\rangle_{E_{3}} \\
& =4\langle 1\rangle+2\left\langle 1, \alpha_{1}, \alpha_{2}, \alpha_{1} \alpha_{2}\right\rangle-2\left\langle 2,2 \alpha_{1}\right\rangle-2\left\langle 2,2 \alpha_{2}\right\rangle-2\left\langle 2,2 \alpha_{1} \alpha_{2}\right\rangle \\
& =4\langle 1\rangle+2\left\langle 1, \alpha_{1}, \alpha_{2}, \alpha_{1} \alpha_{2}\right\rangle-2\left\langle 1, \alpha_{1}\right\rangle-2\left\langle 1, \alpha_{2}\right\rangle-2\left\langle 1, \alpha_{1} \alpha_{2}\right\rangle \\
& =6\langle 1\rangle+2\left\langle\alpha_{1}, \alpha_{2}, \alpha_{1} \alpha_{2}\right\rangle-6\langle 1\rangle-2\left\langle\alpha_{1}, \alpha_{2}, \alpha_{1} \alpha_{2}\right\rangle=0 .
\end{aligned}
$$

Since some of the subgroups are certainly in distinct conjugacy classes the kernel of $h_{L / k}$ is nontrivial so $h_{L / k}$ is not injective.

With these lemmas in hand, the rest of the proof of Theorem 1 is quick. Let $L / k$ be a finite nontrivial Galois extension with Galois group $G$. Suppose that $h_{L / k}$ is injective. Then, by Lemma $9,|G|$ has no odd divisors so $|G|=2^{n}$ for some positive integer $n$. Furthermore, by Lemma $10,|G|=2$ so $G \cong \mathbb{Z} / 2 \mathbb{Z}$ and $L=k(\sqrt{\alpha})$. We know that $G$ has only two subgroups, so, by Proposition 5 , the image of $h_{L / k}$ is generated by linear combinations of $\langle 1\rangle$ and $\langle 2,2 \alpha\rangle$. Since $h_{L / k}$ is injective we have that

$$
a\langle 1\rangle-b\langle 2,2 \alpha\rangle \neq 0 \text { for all } a, b \in \mathbb{Z}^{+} .
$$

It follows from Lemma 8 that $\alpha$ is not a sum of squares, which completes the proof of Theorem 1.

\section{Proofs of Theorem 3 and Lemma 8}

Proof of Theorem 3. Let $L / k$ be a finite Galois extension with Galois group $G$. Suppose that $k$ is quadratically closed in $L$. It follows that $k^{\times} /\left(k^{\times}\right)^{2}$ is finite. 
Clearly, $L \supseteq k\left(\sqrt{\alpha_{1}}, \sqrt{\alpha_{2}}, \ldots, \sqrt{\alpha_{n}}\right)$ where $\left\{\alpha_{1}\left(k^{\times}\right)^{2}, \alpha_{2}\left(k^{\times}\right)^{2}, \ldots, \alpha_{n}\left(k^{\times}\right)^{2}\right\}$ generate $k^{\times} /\left(k^{\times}\right)^{2}$. For every $\alpha_{i}$ there is a subgroup $H_{i} \leq G$ such that $L^{H_{i}}=k\left(\sqrt{\alpha_{i}}\right)$.

If 2 is a square in $k$, then

$$
\begin{aligned}
h_{L / k}\left(G / H_{i}-G / G\right) & =\operatorname{tr}_{L^{H_{i}} / k}\langle 1\rangle_{L^{H_{i}}}-\langle 1\rangle \\
& =\left\langle 2,2 \alpha_{i}\right\rangle-\langle 1\rangle \\
& =\left\langle\alpha_{i}\right\rangle .
\end{aligned}
$$

If 2 is not a square in $k$, then $L \supseteq k(\sqrt{2})$. Let $L^{U}=k(\sqrt{2})$ for $U \leq G$. We have

$$
\begin{aligned}
h_{L / k}\left((G / U-G / G) G / H_{i}-G / G\right) & =\left(\operatorname{tr}_{L^{U} / k}\langle 1\rangle_{L^{U}}-\langle 1\rangle\right) \operatorname{tr}_{L^{H_{i} / k}}\langle 1\rangle_{L^{H_{i}}}-\langle 1\rangle \\
& =(\langle 2,4\rangle-\langle 1\rangle)\left\langle 2,2 \alpha_{i}\right\rangle-\langle 1\rangle \\
& =\langle 2\rangle\left\langle 2,2 \alpha_{i}\right\rangle-\langle 1\rangle \\
& =\left\langle\alpha_{i}\right\rangle .
\end{aligned}
$$

In either case the image of $h_{L / k}$ contains the generators of $G W(k)$ as an abelian group, so $h_{L / k}$ must be surjective.

Now suppose that $h_{L / k}$ is surjective. The composition of $\operatorname{tr}_{L^{H} / k}$ with the functorial map $\mathfrak{r}: G W(k) \rightarrow G W(L)$ gives $\mathfrak{r}\left(\operatorname{tr}_{L^{H} / k}\left(\langle 1\rangle_{L^{H}}\right)\right)=n\langle 1\rangle_{L}$ for any subextension $L^{H}$ and some positive integer $n$ (see [3, §VII 6.4]). Thus, $h_{L / k}$ factors through the group $G W_{L}^{\mathbb{Z}}(k)$ of $k$-quadratic forms $q$ such that $\mathfrak{r}(q)=$ $n\langle 1\rangle_{L}$ for some integer $n$. Since $h_{L / k}$ is surjective, $G W_{L}^{\mathbb{Z}}(k)=G W(k)$, whence $\langle a\rangle_{L}=\langle 1\rangle_{L}$ for all $a \in k^{\times}$. It follows that $k$ is quadratically closed in $L$.

Proof of Lemma 8. Suppose that $a\langle 1\rangle=b\langle 2,2 \alpha\rangle$ where $a, b \in \mathbb{Z}^{+}$. Then the set of elements $D(b\langle 2,2 \alpha\rangle)$ of $k^{\times}$represented by $b\langle 2,2 \alpha\rangle$ and the set of elements $D(a\langle 1\rangle)$ of $k^{\times}$represented by $a\langle 1\rangle$ are equal. Clearly $2 \alpha \in D(b\langle 2,2 \alpha\rangle)$, so $2 \alpha \in D(a\langle 1\rangle)$. It follows that, $2 \alpha$ is a sum of squares in $k^{\times}$. Note that the set of sums of squares in $k^{\times}$is a group under multiplication so $\alpha$ is a sum of squares.

For the other direction we proceed by induction on the stronger claim:

$$
\alpha \text { is a sum of } n+1 \text { squares } \Longrightarrow 2^{n}\langle 1\rangle=2^{n}\langle\alpha\rangle \text {. }
$$

The base case $n=0$ is clearly true. Now assume the claim holds for any sum of $n$ squares. Then, for any sum of $n+1$ squares $\alpha=x_{1}^{2}+\cdots+x_{n}^{2}+x_{n+1}^{2}$, we have, by a standard fact about quadratic forms (see [3, §II 4]),

$$
\left\langle x_{1}^{2}+\cdots+x_{n}^{2}\right\rangle+\left\langle x_{n+1}^{2}\right\rangle=\langle\alpha\rangle\left(\langle 1\rangle+\left\langle x_{n+1}^{2}\right\rangle\left\langle x_{1}^{2}+\cdots+x_{n}^{2}\right\rangle\right) .
$$

If we multiply both sides of the above equality by $2^{n-1}$, it follows from the induction hypothesis that

$$
2^{n-1}\langle 1\rangle+2^{n-1}\langle 1\rangle=2^{n-1}\langle\alpha\rangle\left(\langle 1\rangle+\langle 1\rangle\left\langle x_{1}^{2}+\cdots+x_{n}^{2}\right\rangle\right)
$$

which in turn implies

$$
2^{n}\langle 1\rangle=2^{n}\langle\alpha\rangle
$$


so we have proved the claim.

Now note that for any even positive integer $a$, since 2 is a sum of 2 squares,

$$
a\langle 1\rangle=a\langle 2\rangle .
$$

Putting this together we see,

$\alpha$ is a sum of $n$ squares in $k^{\times} \Longrightarrow 2^{n}\langle 1\rangle=2^{n-1}\langle 2\rangle(\langle 1\rangle+\langle\alpha\rangle)=2^{n-1}\langle 2,2 \alpha\rangle$.

This proves the other direction.

\section{Acknowledgements}

I thank Kyle Ormsby for advising and editing this writeup and Irena Swanson for reviewing an earlier draft. Additionally, I thank the referee for suggesting several helpful improvements to the exposition. I gratefully acknowledge that this research was conducted with support under NSF grant DMS-1406327.

\section{References}

[1] A. W. M. Dress, Notes on the theory of representations of finite groups., Universität Bielefeld, Fakultät für Mathematik, Bielefeld, 1971.

[2] J. Heller, K. Ormsby, Galois equivariance and stable motivic homotopy theory, ArXiv e-prints.Accepted for publication in Transactions of the AMS. arXiv:1401.4728.

[3] T. Y. Lam, Introduction to quadratic forms over fields, Vol. 67 of Graduate Studies in Mathematics, American Mathematical Society, Providence, RI, 2005. 